\title{
An Improved Method of in Vivo Wound Disruption and Measurement
}

\author{
David Charles, M.D., ${ }^{*}$ Kirk Williams, III, B.S., ${ }^{*}$ Larry C. Perry, R.N., $\dagger$ \\ JACK FisheR, M.D., FACS.,† AND RILEY S. REES, M.D., F.A.C.S. \\ *Vanderbilt University School of Medicine, Nashville, Tennessee; †The Reconstructive Surgery Foundation, Nashuille, Tennessee; \\ and $\ddagger$ University of Michigan, Section of Plastic and Reconstructive Surgery, Ann Arbor, Michigan 48109
}

Submitted for publication December 19, 1989

\begin{abstract}
Biomechanical studies of wound strength are important because of new investigations in growth factors, cytokines, and fetal wounds. We compared two traditional methods of wound disruption measurement with a novel computerized model designed for in vivo experiments. An Instron tensiometer (INSTS) and an air insufflated positive pressure device (AIPPD) were compared with a vacuum-controlled wound chamber device (VCWCD). The VCWCD produced vacuum at the wound site and wound disruption was monitored with two video camera/recorders. Rats were marked with a template guide for a $2.5 \mathrm{~cm}$, full-thickness, abdominal incisional wound. Rats were divided into three groups and studied at 2,7 , or 14 days after wounding. The recorded images were computer digitalized to generate wound strength curves from a three-dimensional model. A comparison of the wound disruption curves demonstrated that the VCWCD was comparable to the INST or AIPPD in normal wound healing $(P>.40)$. The VCWCD provided data with less standard error at 2 days after wounding $(P<05)$. In separate series of experiments, VCWCD was tested in the early phases of healing and was found to be sensitive to change at intervals of $48 \mathrm{hr}$ after wounding $(P<.005)$. The INST or AIPPD methods could not perform this task because of an unacceptable level of random error after tissue manipulation. The VCWCD system was considered superior for evaluating early wound healing because it was an in vivo method which required minimal wound manipulation. 1992 Academic Press, Inc.
\end{abstract}

\section{INTRODUCTION}

With new interest in cytokines $[1,2]$, fetal regeneration $[1,3]$, and growth factors $[4,5]$, studies of wound healing are increasingly important. Unfortunately, current methods of wound strength measurement are limited to planimeteric measurement of excised wounds. For example, studies on the biological effects of growth factors require wounds with sufficient tissue strength to secure the tissue and collect the data. Therefore, previous studies with the uniaxial tensiometer $[2,5-7]$ or air insufflated devices $[11,12]$ may be flawed because in vitro techniques require tissue excision. In very early wound healing, excision of the wound disrupts subcutaneous attachments or fibrin deposits which contribute to wound strength [8]. It is possible that early changes in wound strength are not accurately measured since manipulation of fragile wounds introduces a high level of random error. In this study, we compare two tradition measurements of wound healing with a vacuum system modified from techniques described by Mohan [9] and Cook [10]. We present data which suggest that the vacuum device is superior in data collection during the early phases of wound healing.

\section{MATERIALS AND METHODS}

Sprague-Dawley rats $(n=45)$ weighing 250 to $300 \mathrm{~g}$ were divided into three groups and studied at 2, 7, and 14 days after wounding. In separate experiments, the vacuum-controlled wound chamber device (VCWCD) method was used to compare wound disruption in animals ( $n=30$ ) from 1 to 5 days after wounding. Using phenobarbital anesthesia $(45 \mathrm{mg} / 100 \mathrm{~kg}$, intraperitoneally), the abdomen was shaved and prepared. A template was used to mark (Justrite Slink opaque ink) a guide 2.5 $\mathrm{cm}$, for full-thickness, midline abdominal wound. After the wounds were closed with surgical staples (Ethicon), the animals were returned to their cages and fed ad libitum until the wound strength was tested. Prior to any investigations, all surgical staples were carefully removed. At the conclusion of each experiment with the vacuum device, the animals were sacrificed with phenobarbital (125 mg ip). Otherwise, animals were sacrificed prior to study. The animals studies were performed in accordance with the National Research Council's guide for the care and use of laboratory animals.

\section{Vacuum-Controlled Wound Chamber Device (VCWCD)}

A schematic diagram (Fig. 1) of the VCWCD device used for the in vivo wound measurement is depicted. Typically, the wound site was prepared using a template and prominent ink dots were placed adjacent to the surgical 


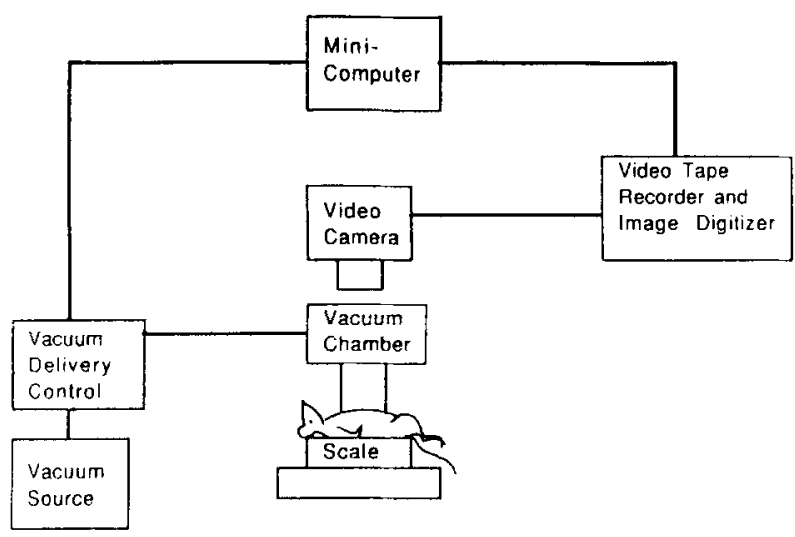

FIG. 1. Schematic diagram of the vacuum control wound chamber device on the rat. The vacuum delivery control, once activated by the minicomputer, reduces the chamber pressure at a constant rate until wound disruption. The video camera(s) record the experiment from the initiation of vacuum until wound disruption. The image digitizer transforms video images to digital data for analysis. The minicomputer reconstructs a three-dimensional model, fully characterizing wound progression to disruption.

incision so that the image digitizer could follow wound disruption. The animal was prepared for the investigation, the vacuum applied until it disrupted, and the chamber pressure fell. All data from each experiment were collected on video tape for statistical analysis.

The computer-assisted image digitizer tracked the ink dots opposite incision on the animal's abdomen (Fig. 2). The acrylic ring was affixed with cyanocrylate glue to the wound site. The ring (i.d. $=2.5 \mathrm{~cm}$ ) defined the area of study and provided a uniform surface for an airtight seal with the vacuum chamber. Once the ring was secured, the animal was placed on the scale (Sartorius
1000MP9), the animal was weighed, and the balance tared. The vacuum chamber was attached to the ring, an airtight seal formed, and the tared balance was maintained at zero weight so that the chamber device applied no stress to the investigations.

The vacuum chamber was a glass cylinder (i.d. $=2.5$ $\mathrm{cm}$ ) enclosed on top with an outlet connecting the vacuum source. The vacuum was applied using a liquid ring high vacuum pump (Nash Corporation, Dalton, GA) which provided a minimum chamber pressure of 22.0 $\mathrm{mm} \mathrm{Hy}$ absulule with a no llow based on $16.7^{\circ} \mathrm{C}$. The vacuum was controlled with a $250 \mathrm{~B}$ pressure/flow controller, a 248A $10000 \mathrm{SV}$ control valve, a 222B 0-1000 torr absolute pressure gauge (9MKS Instruments, Inc., Burlington, MA), and a $\mathbf{4 1 0 . 3 1}$ digital function generator (MTS Corporation, Minneapolis, MN). The MKS equipment provided a closed-loop servo system for vacuum control. The MTS generator controlled the rate of flow and the time the vacuum was applied. Two video cameras (miniature charged-coupled device, Panasonic, Secaucus, NJ, with a 12.5 cosmicar lens) were positioned at different angles over the vacuum chamber to record the skin deformation and disruption in real-time. The scale, vacuum chamber, and video camera were all mounted on a Bencher Illuma System (Bencher 132-30M2) with two quartz lights and motor drive for the camera and chamber. A video character inserter (VICCUR, K-Systems, Inc. Las Cruses, NM) and an Analog-Data Collection System (MP280 with Amdec PC, Motion Analysis Corporation, Santa Rosa, CA) were placed in line with the video camera signal. These two devices recorded on the video tape both experiment conditions and real-time pressure changes in the chamber throughout each experiment.

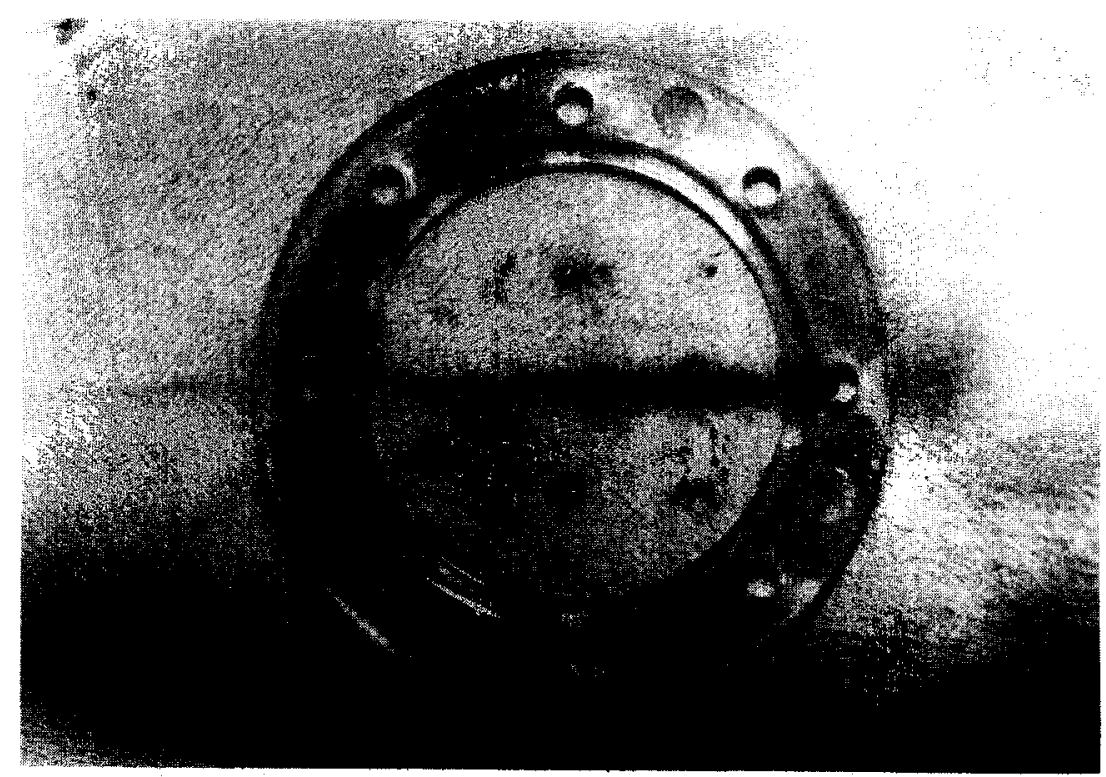

FIG. 2. Demonstrates the acrylic rings placed on the wound in the position viewed by the video cameras. Note the position of the dots opposite the linear wound margin which are all marked with India ink. The wound is $12 \mathrm{hr}$ old. 
Computer analysis of dot movement generated threedimensional models of skin stretch and pressure required to disrupt the wound. The image digitizer (Motion Analysis Corp.) consisted of a video processor (VP310), two videotape recorders (Panasonic AG 6300 $1 / 2$ ), video monitor (Panasonic monochrome), printer (Citoh 3100), Analog-Data Collection System (MP280 with Amdec PC), and Sun Microcomputer (DM2050 [S1] graphics work station, 3/100 CPU, dual 71 megabyte tape drives).

The VCWCD allowed precise measurement of wound bursting, breaking strength, and skin deformation. The computer analysis of the recorded images and vacuum pressure/analog recordings produced real-time data files during application of the vacuum. Stress/strain curves were generated for extended calculations of the biomechanical properties of modulus of elasticity and total energy absorption. The pressure (stress) and deformation (strain) files were merged to generate stress/strain curves for each corresponding skin characterization analysis. The $\mathrm{Y}$-axis was displayed with pressure (units $=\mathrm{KPa}$ ), and the $\mathrm{X}$-axis representing the displacement of the dots (units $=\mathrm{mm}$ ). The modulus (E) was calculated from the most linear portion of the slope, and the energy absorption (U) was computed from the area under the curve. Upon completion of each raw data analysis, the computer automatically stored the results for statistical analysis.

\section{Instron Tensiometer (INST)}

The wound breaking strength was measured using a tensiometer (Instron Model 5100). Briefly, the abdominal skin containing the wound was excised carefully from the sacrificed animal to avoid introducing distortion in the study area. The tissue was placed in the clamps of the INST and progressive force was applied until the wound disrupted. The breaking strength was calculated based on the grams of force required to break the wound.

\section{Air Insufflated Positive Pressure Device}

The wound bursting strength was measured using an air insufflated positive pressure device (AIPPD) used by Myers [7]. Briefly, the abdominal wounds were carefully excised with sharp dissection to avoid distortion of the study area. The excised wounds were secured on a compressing O-ring overlying a $2 \times 1 \mathrm{~cm}$ hole in the top of a cylindrical pressure chamber. The air was insufflated into the chamber until the wound disrupted and the pressure required to break the wound recorded in pounds per square inch using an in-line gauge connected through the insufflation port.

\section{Statistical Significance}

The data generated from the VCWCD and AIPPD methods were expressed as pounds per square inch while data generated from the INST method were expressed in

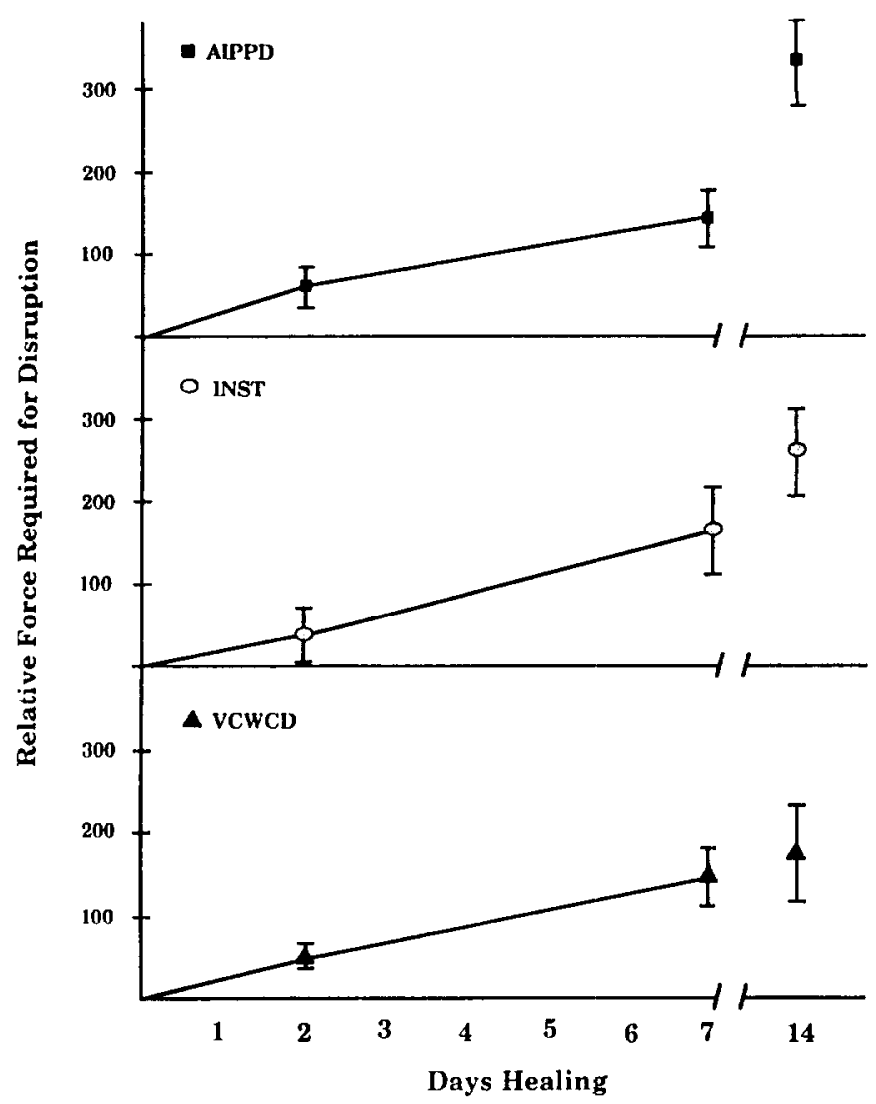

FIG. 3. Comparison of the wound disruption curves for each method. The data is normalized to allow a standardized comparison of the three methods. The air insuffalated positive pressure device (AIPPD), instron tensiometer (INST), and the vacuum control wound chamber device (VCWCD) produce similar wound healing curves during the first week of wound healing $(P>0.40)$.

grams per cross sectional area. Therefore to compare the three methods, the data was normalized as percentage control and expressed as the mean and standard deviation from the mean. One-way analysis of variance was used to determine if any of the three methods provided statistically different wound healing curves.

In the second series of experiments, the data collected for the VCWCD method for Days 1 through 5 were analyzed with a one-way analysis of variance using an " $F$ " test for equality of varience. The differences in standard error were compared in the three groups and significance defined as $P<.05$.

\section{RESULTS}

In the first series of experiments, we compared the three methods of wound disruption at 2,7 , and 14 days post wounding. There was good correlation between all three experimental methods and the slope of the curves was similar (Fig. 3). There was no statistical significant difference $(P>0.40)$ between the slopes of the lines in any of three methods used. Studies were not performed after 14 days because the VCWCD method generated insufficient vacuum force to burst the wounds. 
TABLE 1

A Comparison of Wound Strength Measurement Using Different Techniques at 2 Days Postwounding

\begin{tabular}{clll}
\hline Observations & VCWCD $^{a}$ & AIPPD $^{a}$ & INST $^{b}$ \\
\hline 1 & 1.5 & 2.1 & 3.0 \\
2 & 1.1 & 2.9 & 0.9 \\
3 & 1.6 & 1.5 & 1.6 \\
4 & 1.5 & 3.0 & 1.5 \\
5 & $\underline{1.4}$ & $\underline{2.0}$ & $\underline{4.6}$ \\
Means \pm SD* $^{*}$ & $1.42 \pm 0.19$ & $2.3 \pm 0.63$ & $2.48 \pm 1.62$ \\
\hline
\end{tabular}

Note. The data were normalized as a percentage of mean value.

${ }^{a}$ Required eight animals for five observations.

${ }^{b}$ Required eight animals for five observations.

${ }^{*} P<0.05$. SD, standard deviation.

The 2-day-old wounds were friable and fell apart during dissection and we interrupted these observations as evidence that the VCWCD measurement was much more sensitive since the studies were done in vivo. When the techniques from each group were prepared for the mean and standard error of the mean, the data suggested that the VCWCD group had less standard error in wound measurement than the other techniques (Table 1). This data was statistically significant using an $F$ test and ANOVA $(P<.05)$. The data in Table 1 demonstrate the great variability in wound strength results with INST or AIPPD methods.

To pursue this observation further, we sought to determine if the VCWCD was effective in detecting differences in wounds strength immediately after wounding and the subsequent 5 days thereafter. In fig. 4 , the data demonstrates that the VCWCD method showed increased wound strength during each day after wounding. These differences are statistically significant $(P<.05)$ except Days 2 and 3. We interrupted this data as evidence that the wound strength differences could be detected in vivo within 1 day following wounding.

A unique attribute of the VCWCD system was the ability to measure the modulus of elasticity during data collection for bursting strength. (Table 2). In a typical experiment, the percentage that the standard deviation from the mean value of bursting strength, skin deformation, and modulus of elasticity was approximately $13 \%$. Using equality of variance, this data suggested that there was a statistically significant correlation between these the variables in wound healing $(P<.05)$. However, at 2 days the modulus of elasticity didn't correlate with the other variables. These observations suggested the elasticity of the wound and bursting strength were not directly related in early wound healing.

\section{DISCUSSION}

In this study, we have compared a novel wound strength measurement with standard tensiometer and

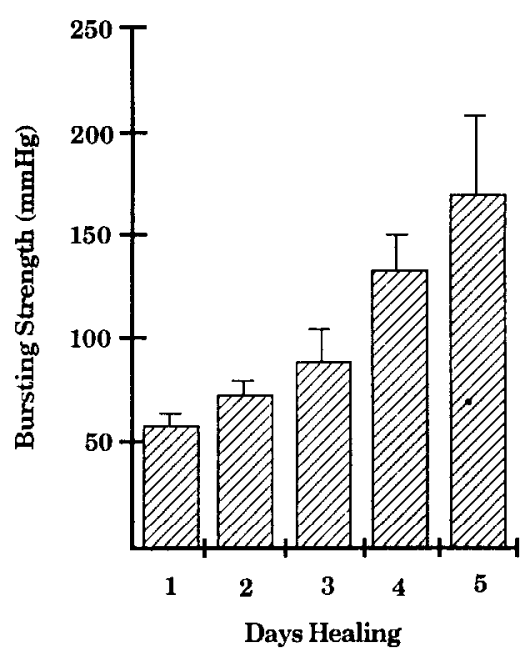

FIG. 4. The bursting strength for Days 1 through 5 postwounding using the vacuum control wound chamber device. All days were statistically significantly different $(P<0.05)$ from each day except for the differences between Day 2 and Day 3. The data were expressed in $\mathrm{mm}$ $\mathrm{Hg}$.

pressure chamber measurements. Our system uses sophisticated computer technology, high speed video recording, and analysis of three-dimensional motion to measure proportional wound strength over time. Computer analysis of the video tape-recorded experiments generate time, pressure, and wound deformation curves during disruption. Although the system requires elaborate equipment for data acquisition and interpretation, the actual technique is simple and reproducible.

Despite the complexity of the VCWCD system, there are significant advantages. The unique method allows the investigator to study wounds in vivo and in situ which is not possible with other methods. This attribute is applicable to immature wounds or fragile fetal wounds where tissue manipulation produces wide standard error.

With the aid of a computer program, the modulus and energy absorption are determined from the linear portion of the corresponding stress/strain curve. After selection of the respective points, each parameter is auto-

\section{TABLE 2}

Typical Experiment Demonstrating a Comparison of Bursting Strength, Skin Deformation, and Modulus of Elasticity

\begin{tabular}{|c|c|c|c|c|c|c|c|}
\hline \multirow[b]{2}{*}{ Variable } & \multirow[b]{2}{*}{$n$} & \multicolumn{3}{|c|}{ Day 2} & \multicolumn{3}{|c|}{ Day 7} \\
\hline & & Mean & $\mathrm{SD}$ & $\%$ Mean $^{a}$ & Mean & SD & \%Mean \\
\hline Strength (mm Hg) & 5 & 68.02 & 6.93 & $10 \%$ & 246.00 & 32.38 & $13 \%$ \\
\hline Deformation (mm) & 5 & 4.76 & .51 & $11 \%$ & 4.06 & 0.62 & $13 \%$ \\
\hline Modulus $(\mathrm{kPa})^{b}$ & 5 & 36.76 & 6.28 & $17 \%$ & 214.00 & 29.79 & $14 \%$ \\
\hline
\end{tabular}

a Mean, Indicates the percentage that the standard deviation is from the mean value.

${ }^{b} \mathrm{kPa}$, kilopascals. 
matically calculated and stored for statistical analysis. Currently, there is no biomedical method available to measure these parameters.

Comparison of wound strength measurements between INST, AIPPD, and VCWCD is difficult because INST measures breaking strength while AIPP and VCWCD measure bursting strength. Breaking strength is a measure of a one-dimensional force required to break an excised wound along its dimensions [11]. Bursting strength is the measure of a three-dimensional force that causes a spherical deformation required to break the wound regardless of its dimensions.

The VCWCD system is a combination of two wound disruption models described by Mohan [9] and Cook [10]. Cahill [12] showed that the combination of these techniques allowed for the mathematical calculation of not only bursting strength but also the extension ratio, tissue elasticity (modulus), and breaking strength. Therefore, the VCWCD is able to determine both bursting and breaking strength as described by Peacock [11].

Presently the prototype device with the computer and video equipment is half of the total cost for an INST. However, as subsequent models are developed and technology changes, this cost may reduce significantly. The entire system except for the vacuum supply is contained within 100 square feet of laboratory space. The vacuum supply has two very large tanks occupying 200 additional square feet. An enhanced vacuum source would not be required for most applications of the VCWCD such as the study of early wounds or fragile tissue. The VCWCD is far superior to the other methods of study when evaluating early wounds or fetal wounds.

\section{ACKNOWLEDGMENTS}

M. Burt Myers, M.D., provided his model of a pneumatic tensiometer without which this project would not have been complete. This project was supported by the Department of Veterans Affairs.

\section{REFERENCES}

1. Krummel, T. M., Michna, B. A., Thomas, B. L., Sporn, M. B., Nelson, J. M., Salzberg, A. M., Cohen, I. K., and Diegelman, R. F. Transforming growth factor $\beta$ (TGF- $\beta$ ) induces fibrosis in a fetal wound model. J. Pediatr. Surg. 23: 647, 1988.

2. Mustoe, T. A., Pierce, G. F., Thomason, A., Gramates, P., Sporn, M. B., and Deuel, T. F. Accelerating healing of incisional wounds in rats induced by transforming growth factor $-\beta$. Science $\mathbf{3 3 7}$ : $1333,1987$.

3. Krummel, T. M., Nelson, J. M., Diegelmann, R. F., Lindbald, W. J., Salzberg, A. M., Greenfield, L. J., and Cohen, I. K. Fetal response to injury in the rabbit. J. Pediatr. Surg. 22: 640, 1987.

4. Brown, G. L., Nanney, L. B., Griffen, J., Cramer, A. B., Yancy, J. M., Curtsinger, L. J., Holtzin, L., Schultz, G. S., Jurkiewicz, M. J., and Lynch, J. B. Enhancement of wound healing by topical treatment with epidermal growth factor. N. Engl. J. Med. 321: 76, 1989 .

5. Brown, G. L., Curtsinger, L. J., White, M., Mitchel, R. O., Pietsch, J., Nordquist, R., Von Franhofer, A., and Schultz, G. Acceleration of tensile strength of incisions treated with EGF and TGF- $\beta$. Ann. Surg. 208: 788, 1988.

6. Crawford, D. T., Bains, J. W., and Ketcham, A. S. A standard model for tensiometric studies. J. Surg. Res. 6: 265, 1965.

7. Myers, M. B., Cherry, G., Heimburger, S., Hay, M., Haydel, H., and Cooley, L. The effect of edema and external pressure on wound healing. Arch. Surg. 94: 218, 1967.

8. Rovee, D. T., and Miller, C. M. Epidermal role in the breaking strength of wounds. Arch. Surg. 96: 43, 1968.

9. Mohan, I., and Melvin, J. Failure properties of passive human aortic tissue. II. Biaxial tension test. J. Biomech. 16: 31, 1983.

10. Cook, T., Alexander, H., and Cohen, M. Experimental method for determining the 2-dimensional mechanical properties of living human skin. Med. Biol. Eng. Comput. 15: 381, 1977.

11. Peacock, E. E., and Van Winkle, W., Jr. Wound Repair. Philadelphia: Saunders, 1984. Pp. 105-107.

12. Cahill, B. P., An, K. N., Fisher, J., and Chao, E. Y. S. In vivo test system for characterization of skin wound healing. Biomed. Sci. Instr. 23: 29, 1987. 